\title{
Validity of Self-Reported Diabetes in a Cohort of Thai Adults
}

\author{
Keren Papier ${ }^{1,2}$, Susan Jordan ${ }^{2}$, Chris Bain ${ }^{1,2}$, Catherine D’Este ${ }^{1}$, Prasutr Thawornchaisit ${ }^{3}$, \\ Sam-ang Seubsman ${ }^{4} \&$ Adrian Sleigh ${ }^{1}$ \\ ${ }^{1}$ National Centre for Epidemiology and Population Health (NCEPH) and Department of Global Health, Research \\ School of Population Health, College of Medicine, Biology and Environment, The Australian National University, \\ Canberra, Australia \\ ${ }^{2}$ Population Health Department, QIMR Berghofer Medical Research Institute, Brisbane, Australia \\ ${ }^{3}$ Lerdsin General Hospital, Bangkok, Thailand \\ ${ }^{4}$ Thai Health-Risk Transition Study, School of Human Ecology, Sukhothai Thammathirat Open University, \\ Nonthaburi, Thailand \\ Correspondence: Keren Papier, QIMR Berghofer Medical Research Institute, 300 Herston Rd, Brisbane, 4006 \\ QLD, Australia. Tel: 61-7-3845-3571. E-mail: Keren.Papier@anu.edu.au
}

Received: September 30, 2016 Accepted: December 13, 2016 Online Published: December 14, 2016

doi:10.5539/gjhs.v9n7p1 URL: http://dx.doi.org/10.5539/gjhs.v9n7p1

\begin{abstract}
Background: Much of South East Asia is experiencing an epidemiological transition. In Thailand, chronic disease has emerged and the prevalence of diabetes has tripled. As part of a large cohort study of the Thai transition to chronic disease, we gathered data on self-reported diabetes. Epidemiological studies commonly ascertain such data by self-report but the validity of this method has not been assessed in Thailand. Therefore, we aimed to investigate the validity of self-reported type 2 diabetes (T2DM) in Thai adults participating in the Thai Cohort Study (TCS).
\end{abstract}

Methods: Data were collected by mailed questionnaire from adults involved in the TCS, a nationwide community-based longitudinal health study of distance learning adult students enrolled at Sukhothai Thammathirat Open University. Participants were surveyed in 2005, 2009 and 2013. We sampled all participants with self-reported T2DM status (878 cases) for telephone interview with our study physician along with a random selection of 650 participants who self-reported not having diabetes in all three TCS surveys. These physician telephone interviews allowed us to validate self-reported questionnaire responses.

Results: Questionnaire self-report of diabetes slightly over-estimated the incidence of T2DM in this cohort; the overall proportion of confirmed T2DM cases was $78 \%$. Participants with a consistent pattern of diabetes reporting at the 2009 and 2013 questionnaire follow-ups had the highest validity of self-reported responses $(96 \%$; $95 \% \mathrm{CI}$ 92.9-99.1).The lowest proportion of confirmed T2DM cases was recorded among participants who reported diabetes in 2009 and not in 2013 (32\%)(95\%CI 22.6-41.4), mostly young women with transient (gestational) diabetes.

Conclusions: Our results, derived mainly from young, educated Thai adults nationwide, show that self-reported doctor diagnosed T2DM is a feasible and acceptable method for assessing diabetes in epidemiological studies.

Keywords: cohort study, diabetes mellitus, type 2, self-reported diabetes, Thailand, validation study

\section{Introduction}

Many developing countries have undergone rapid economic growth over the past 50 years and this has transformed diets, behaviors and disease. There have been great health benefits associated with this transition, including decreased childhood mortality and reduced prevalence of infectious diseases. But there has also been a widespread adoption of unhealthy behaviors (such as smoking and lack of exercise), along with a concomitant emergence of unhealthy environments (such as urban slums and air pollution), and a transformation of food systems and agrarian diets into modern supermarkets and industrialized food. These changes lead to the emergence of chronic and degenerative diseases. Collectively, these shifts in behavior, environment, diet, and disease have been labelled the 'health-risk transition' (A. Sleigh \& Seubsman, 2015). As part of the transition, T2DM has emerged as a major cause of morbidity in many middle-income countries, including Thailand (Ramachandran, Wan Ma, \& Snehalatha, 
2010). With over 4.0 million Thai adults estimated to have diabetes, Thailand is now one of the most affected countries in Asia (Chan et al., 2009; International Diabetes Federation, 2015).

To respond to the emerging non-communicable disease epidemic in Thailand, and understand the local risk factor dynamics, researchers from Thailand and Australia have established the 'Thai Cohort Study', a nation-wide investigation of the ongoing 'health-risk transition' (A. C. Sleigh, Seubsman, Bain, \& The Thai Cohort Study Team, 2008). Like many large-scale epidemiological studies, the TCS used self-completed questionnaires to collect information on risk factors and disease, including diabetes. Collecting health information via self-report is a feasible and convenient method for obtaining population data but questions arise over accuracy of this method. Responses by study participants may vary depending on their personal characteristics, including education level and perceptions and understanding of disease (Goto et al., 2013; Okura, Urban, Mahoney, Jacobsen, \& Rodeheffer, 2004).

Several studies have suggested that a diagnosis of diabetes is accurately reported by study participants however this work was carried out in cohorts restricted to women (Manson et al., 1991; Pradhan, Manson, Rifai, Buring, \& Ridker, 2001; Rylander, Sandanger, Engeset, \& Lund, 2014) or people within a specific age range (Comino et al., 2013; Goldman, Lin, Weinstein, \& Lin, 2003; Margolis et al., 2008). Among studies conducted within larger or more heterogeneous cohorts, the accuracy of self-reported diabetes varied by socio-demographic characteristics (Okura et al., 2004; Yuan, Liu, Wu, Zou, \& Li, 2015) or by ethnicity (El Fakiri, Bruijnzeels, \& Hoes, 2007; Goto et al., 2013). Socio-demographic characteristics such as older age (with cognitive decline) (Sherbourne \& Meredith, 1992) and lower education level (with reduced health literacy) (Yuan et al., 2015) may associate with reduced accuracy of self-reported diabetes. Little research has assessed the accuracy of self-reported health information among Asian populations, although it has been suggested that there may be higher levels of misreporting than in western populations (Goldman et al., 2003; Goto et al., 2013; Yuan et al., 2015).

Accuracy of self-reported health status among Asian populations may link to traditional cultural beliefs. In some Asian countries (including China and Thailand), traditional medicine may be practiced alongside and/or as part of the healthcare system (Chokevivat, Chuthaputti, \& Khumtrakul, 2005; Hesketh \& Zhu, 1997). Treatment for health conditions may be sought using traditional and/or modern medicine approaches (Yuan et al., 2015). As a result, traditional medical perspectives and/or treatment may lead to misreporting or under-reporting in Asian populations. For example, individuals using traditional Chinese medicine may under-report hypertension (Goldman et al., 2003) since high blood pressure may not be considered as a disease according to traditional Chinese medicine (Goldman et al., 2003). In contrast, Chinese adults self-report diabetes more accurately when also questioned about use of traditional Chinese anti-diabetic medicine (Yuan et al., 2015). Whether similar issues are relevant in Southeast Asian populations is not known. Specifically, the validity of diabetes self-report has not been investigated in the Thai population. Many Thais practice traditional Thai medicine which is highly influenced by both Theravada Buddhism (the main school of Buddhism practiced in Thailand) and by traditional Chinese medicine (Offringa, 2014). Accordingly, field studies of self-reported diabetes among Thais may be affected by traditional cultural beliefs just as noted in Chinese populations and this might influence the epidemiological information.

Therefore, we validated questionnaire self-report of doctor-diagnosed T2DM in Thai adults participating in the TCS by comparing physician interview data to the questionnaire data. We also investigated whether the validity of self-reported questionnaire T2DM was associated with personal socio-demographic characteristics. We then determined the impact that any potential reporting bias may have on cumulative incidence estimates for T2DM.

\section{Methods}

\subsection{Source Population}

In 2005 all 200,000 enrolled Suhkkothai Thammathirat Open University (STOU) students were mailed a detailed baseline questionnaire that covered a wide range of topics including socio-demographic characteristics, lifestyle behaviors and self-reported health outcomes. These students were adult distance learning students of modest means aspiring to use education for self-improvement. As such they are expected to undergo the 'health-risk transition' dynamics ahead of their fellow Thais. Of the total 87,151 (100\%) students who returned their questionnaire in 2005, 60,569 (69\%) were successfully followed up in 2009 and $42,785(49 \%)$ were followed up again in 2013.

\subsubsection{Ascertainment of Diabetes Status}

In all three surveys (baseline, four-year follow-up, and eight-year follow-up), cohort members were asked whether they had ever been told by a doctor that they had diabetes. The questionnaire did not ask participants to specify the 
type of diabetes (Type 1, Type 2, gestational, etc). Therefore all those who ticked 'yes' to this question have been classified as self-reported cases of diabetes mellitus (with the type of diabetes not specified). This included a small group who reported 'yes' in 2009 but reverted to 'no' at follow-up in 2013.

\subsubsection{Participant Selection for the Validation Study}

Figure 1 shows how participants were selected for this validation study. We excluded the 902 participants in the original TCS cohort who reported doctor-diagnosed diabetes at baseline (2005). Exclusion of baseline prevalent cases ensured our focus was on current diagnostic practices, as incident cases (reported in 2009 and 2013) were of recent onset and would capture outcomes of the 'health-risk transition'. Of those not excluded, participants who completed both follow-up questionnaires $(2009,2013)$ were sampled for the current study. This allowed us to assess whether an individual's sequential reporting pattern over the 2009 and 2013 follow-up surveys influenced their likelihood of a valid self-reported diagnosis. Those who reported doctor-diagnosed diabetes for the first time in either of the follow-up questionnaires were considered as self-reported incident cases for our study and all 878 were included in the validation sample. Those who reported not having diabetes in all three of the TCS surveys $(2005,2009,2013)$ were considered non-cases $(n=38,809)$, of whom we randomly sampled 650 .

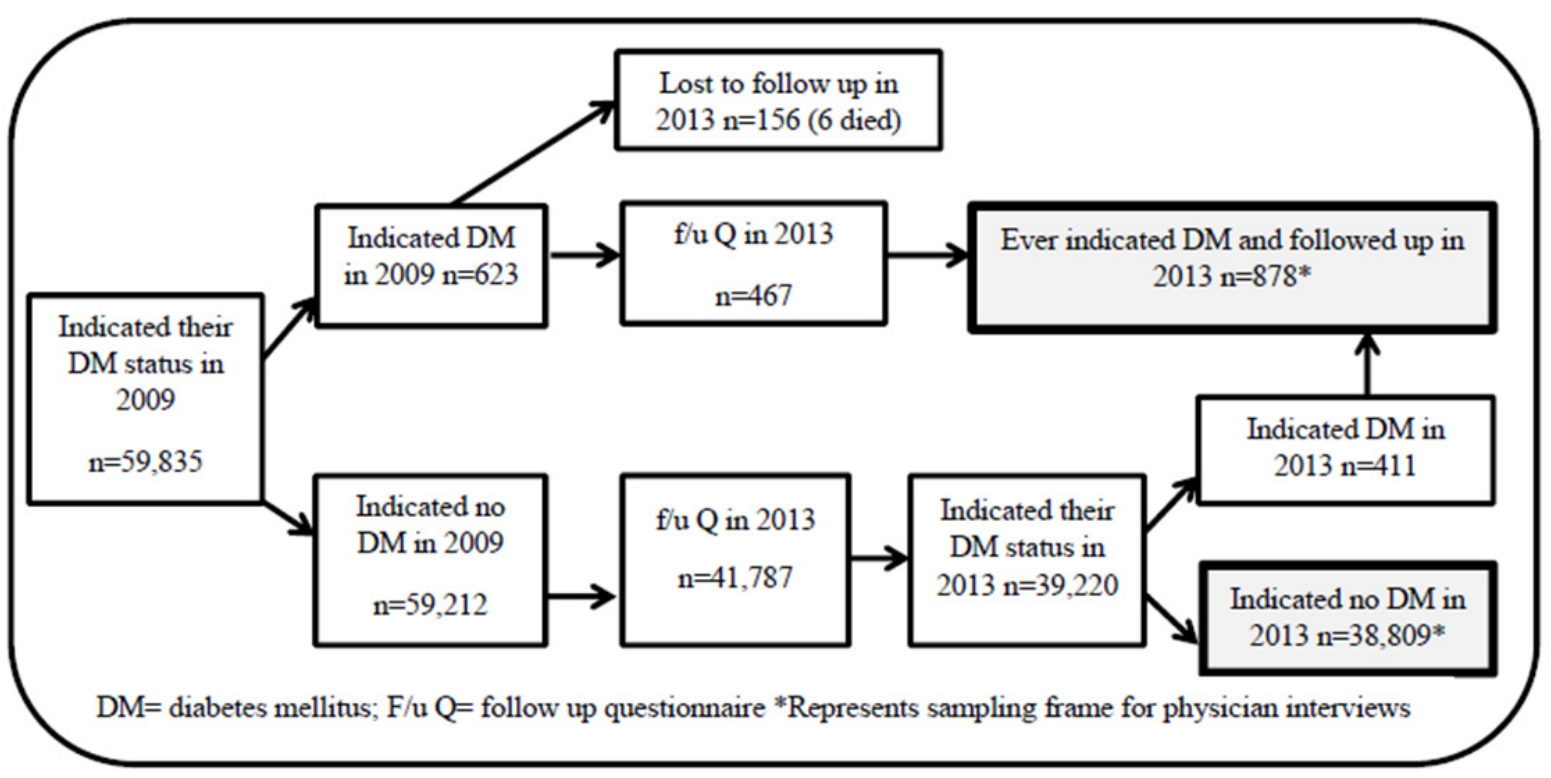

Figure 1. Ascertainment of diabetes cases in Thai Cohort Study participants

Participants* selected based on self-reported diabetes status in 2005, 2009 and 2013.

*The self-reported positives in 2005 were excluded $(\mathrm{n}=902)$ as were those missing in 2009 . The 59,835 remaining persons were subjected to the sequential process involved in the validation and the numbers of persons in each category as shown in the figure above.

\subsection{Data Collection}

We used telephone interviews undertaken by a practicing Thai physician to confirm the validity of self-reported doctor-diagnosed T2DM and transient diabetes. Medical record review and/or blood sampling for this nationally dispersed cohort was not logistically feasible. Furthermore, blood measures alone can be uninformative or even misleading for those with diabetes who are receiving treatment and whose blood glucose levels have normalized.

The same Thai physician conducted all interviews to exclude the potential for variation between interviewers. He was selected because of his previous experience with eliciting medical information from the TCS cohort, his knowledge of the use of traditional Thai diabetes medication, and his sensitivity to the culturally specific language required to attain information about such usage.

\subsubsection{Interview Procedure for Validation Study}

Potential participants were first sent an information sheet describing the validation study and inviting their participation before they were telephoned. The information emphasized that participation was not compulsory and it would be entirely their choice to take part or not. Then, up to three attempts were made to telephone each person 
over a 6 week period between May and June 2015. The physician received verbal consent from each interviewee before conducting the interview. After receiving consent, the physician interviewed the sampled participants and progressively characterized each person according to the algorithm in Figure 2.

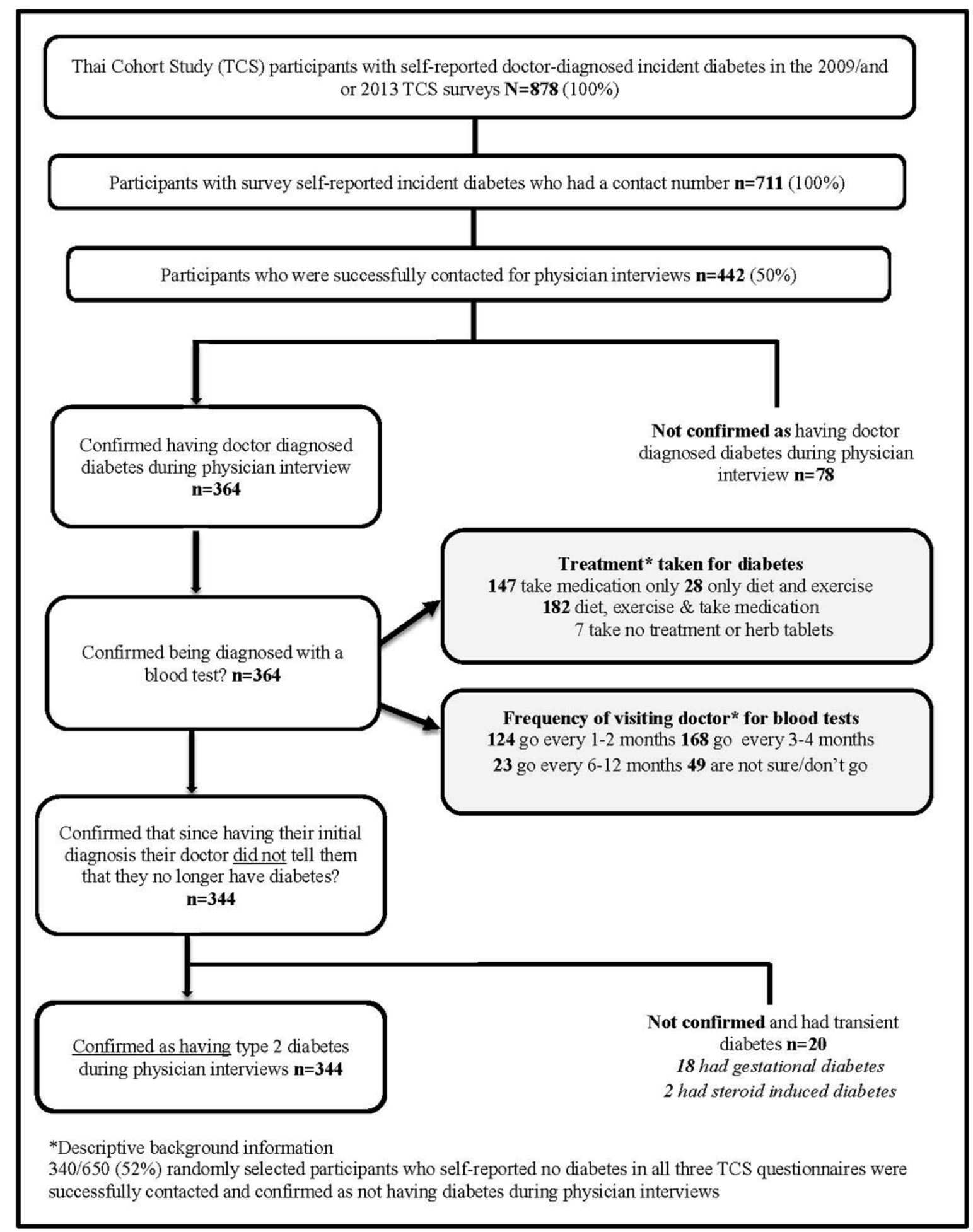

Figure 2. Algorithm used to determine type 2 diabetes status during physician interviews

Physician interviews* were used to determine type 2 diabetes status among sampled participants.

Cases and non-cases were determined using an interview protocol and using the above algorithm. 


\subsubsection{Ascertainment of Diabetes Cases Based on Physician Interviews}

Type 2 diabetes status was determined by an algorithm incorporating the participants' answers to a standard physician interview protocol. The protocol was developed by a group of five physicians (four Australian and one Thai) and two public health nutritionists, following the American Diabetes Association's (ADA) classification guidelines for diagnosing diabetes (American Diabetes Association, 2010). The ADA guidelines are the most commonly used criteria for diagnosing diabetes globally (Shaw, Sicree, \& Zimmet, 2010) and are used by researchers and health professionals in Thailand (Aekplakorn et al., 2011). Accordingly, the protocol included questions probing about blood glucose testing and if it had occurred and blood glucose cut off criteria used in the participants' diagnoses of diabetes, as well as the treatment and names of medications prescribed to participants by their physician. The interviewer asked about the frequency of physician check-ups and the types of tests taken during medical visits. Finally, those reporting diabetes were questioned further about transient disease triggered by pregnancy, surgery, use of steroids or other factors. The algorithm used for ascertaining T2DM status based on participants' answers is shown in Figure 2.

\subsection{Statistical Analysis}

Participants who self-reported diabetes were classified firstly as having correctly reported a diagnosis of diabetes mellitus. They were then classified according to whether or not they had T2DM or transient diabetes based on interviews by our study physician as outlined in Figure 2. It was noted if the transient diabetes was due to pregnancy, surgery, medication, or illness. The sample of participants who self-reported no diabetes in all three surveys were also interviewed by the study physician to confirm their status.

\subsubsection{Validation of Self-Reported Questionnaire Data}

Amongst the contacted participants, we calculated the proportion of valid self-reported questionnaire responses by dividing the number of physician interview-confirmed T2DM cases by the total number of participants who self-reported diabetes in 2009 and/or 2013. We also confirmed the proportion of validated non-cases by dividing the number of physician interview-confirmed non-cases by the total number of participants who self-reported not having diabetes in all three of the TCS surveys. We then assessed whether the proportion of physician-interview confirmed T2DM cases varied according to various socio-demographic characteristics previously found to be associated with the accuracy of self-reporting diabetes (Molenaar, Van Ameijden, Grobbee, \& Numans, 2007; Okura et al., 2004).

We wanted to assess which factors were associated with the correct self-reporting of T2DM separately for self-reported cases and self-reported non-cases. To do this we undertook logistic regression analysis including only the self-reported cases to investigate the socio-demographic characteristics associated with self-reported questionnaire and physician telephone interview agreement (with a binary outcome of no/yes for agreement). A likelihood ratio test was used to assess the significance of the variables in the model. A two sided significance level of 0.05 was used. The regression model included all physician interview confirmed T2DM cases. We then repeated these analyses including all of the physician-interview-confirmed diabetes cases (both T2DM and the transient diabetes cases).

We then investigated how the sequential reporting pattern over the 2009 and 2013 follow-up surveys influenced the likelihood of a valid self-reported diagnosis of T2DM in 2013. Accordingly, we calculated the proportion of validated cases amongst 1) those who self-reported incident diabetes in both 2009 and 2013;2) those who reported diabetes for the first time in 2013; and 3) those who reported diabetes in 2009 and subsequently reported no diabetes in 2013.

\subsubsection{Incidence of T2DM Accounting for the Effect of Misclassification}

We also determined the impact that reporting error may have had on estimates of cumulative incidence of T2DM in the cohort. A corrected cumulative incidence was calculated in two ways. We used the proportion of reporting error detected in 2009 incident cases amongst the contacted group to calculate a corrected cumulative incidence among the entire group of 2009 self-reported cases including those who did not participate in the interviews. The same procedure was carried out for the entire group of 2013 self-reported cases using the proportion of error detected in the 2013 self-reported cases who were contacted.

We also carried out a sensitivity analysis to allow for the possibility that the amount of reporting error might be different among the group of participants who were lost-to-follow-up and thus did not participate in the telephone interviews. In this analysis we considered the effects of false positive probabilities by calculating a corrected cumulative incidence for the entire group of 2009 self-reported cases using false positive probabilities ranging between $10 \%$ and $50 \%$ for participants who did not participate in the interviews and were lost-to-follow up. All 
analyses were carried out using Stata (version 13.0).

\subsubsection{Sample Size, Power, Precision}

Sample size consideration for this study was guided by findings from previous validation studies conducted with Asian cohorts (Goto et al., 2013; Yuan et al., 2015). We expected that half of the selected cohort members who the physician attempted to contact would be reached by phone and would agree to take part in the interview (Kelly, Seubsman, Banwell, Dixon, \& Sleigh, 2014). Accordingly, of the total 878 self-reported diabetes cases we expected to successfully follow-up approximately 440. A sample of 440 participants with diabetes would allow estimation of an expected correct reporting proportion of $80 \%$ (Goto et al., 2013; Yuan et al., 2015) with 95\% confidence interval within $+/-4 \%$. With a higher proportion of correct reporting expected among non-cases (85\%), a smaller sample of 325 participants without diabetes would allow for the estimation of $95 \%$ confidence interval within the same precision of $+/-4 \%$.

Of the 442 self-reported cases, we expected that $80 \%$ (354 participants) would be verified as having T2DM and that $20 \%$ (88 participants) would not be verified as having T2DM. Thus, using an expected reporting error of $20 \%$, $80 \%$ power and $5 \%$ significance level, we would be able to detect a $15 \%$ difference in socio-demographic characteristics between those who were and were not confirmed as correctly self-reporting T2DM and between participants who were and were not confirmed as having correctly self-reported diabetes (both T2DM and the transient diabetes cases) during physician interviews.

\section{Results}

Table 1 displays the baseline characteristics of the self-reported cases and non-cases that were selected for the physician telephone interviews. Among the self-reported cases, 711 of 878 had contact phone numbers and among the 650 non-cases 616 had contact phone numbers and were invited to participate. Of the participants who were selected for interviews, $442(50 \%)$ self-reported cases and 340 (52\%) self-reported non-cases were successfully contacted for interview. All participants with whom the physician made contact participated in the study. Among the contacted cases, the median age at baseline was 39.5 (minimum 19, maximum 64) and 52\% were male. Among the contacted non-cases just over half were female (54\%), and their median age at baseline was 31.5 (range 18 to 78).

Overall the socio-demographic characteristics of those interviewed and those who could not be contacted were similar for self-reported cases and for non-cases (all p-values $>0.05$ ). Differences were observed between cases who were interviewed and cases who were not interviewed for monthly income level $(p=0.02)$ (higher income in those interviewed) and between non-cases who were interviewed and non-cases who were not interviewed for age $(\mathrm{p}=0.02)$ (lower age in those interviewed).

The physician interviews confirmed that 344 of the 442 (78\% (95\%Confidence Interval (CI)) 74-82\%) contacted cases reporting a new diagnosis of diabetes in either the 2009 or 2013 surveys had incident T2DM (shown in figure 2). Of the 98 cases that were not confirmed as having T2DM, twenty percent were found to have had transient diabetes mellitus (18 gestational and two steroid-induced diabetes). The majority of these transient cases self-reported diabetes in 2009 but not in 2013. Of the remaining 78 non-confirmed cases, the majority indicated they self-reported diabetes because they were told by their physician that they had high blood glucose and were at risk of developing diabetes. The other participants indicated that they had misunderstood the questionnaire and thought that it was asking if they had ever been tested for diabetes. All $340(100 \%)$ participants who indicated in both 2009 and 2013 that they had not been diagnosed with diabetes were found to have reported their disease status correctly.

The proportions of self-reported diabetes cases validated by physician interviews according to various socio-demographic characteristics are shown in Table 2. The overall proportion of all confirmed self-reported diabetes cases (including participants with T2DM and with transient diabetes) was high (82\%) and was similar across all socio-demographic characteristics. The overall proportion of confirmed self-reported T2DM diabetes cases was high (78\%). Slight differences in the proportion of confirmed cases of T2DM were seen between males and females $(82 \%$ versus $71 \% \mathrm{p}<0.01)$ and between participants aged over 40 and those aged under $40(84 \%$ versus $72 \% \mathrm{p}<0.01)$. 
Table 1. Baseline characteristics for participants and non-participants selected for physician interviews to validate self-reported diabetes status

\begin{tabular}{|c|c|c|c|c|c|c|}
\hline \multirow{4}{*}{ Baseline characteristics } & \multicolumn{3}{|c|}{ Ever reported incident diabetes } & \multicolumn{3}{|c|}{ Never reported incident diabetes } \\
\hline & Participants & Non-participants & & Participants & Non-Participants & \\
\hline & & & $P^{\dagger}$ & & & $P^{t}$ \\
\hline & $\mathrm{n}(\%)$ & $\mathrm{n}(\%)$ & $P$ & $\mathrm{n}(\%)$ & $\mathrm{n}(\%)$ & \\
\hline Sex & & & 0.29 & & & 0.48 \\
\hline Male & $282(52)$ & $263(48)$ & & $143(51)$ & 139 (49) & \\
\hline Female & $160(48)$ & $173(52)$ & & $197(54)$ & $171(46)$ & \\
\hline Age & & & 0.14 & & & 0.02 \\
\hline $15-29$ & $58(41)$ & $82(59)$ & & $147(47)$ & $163(53)$ & \\
\hline $30-39$ & $168(53)$ & $149(47)$ & & $118(54)$ & 101(46) & \\
\hline $40-49$ & $158(51)$ & $150(49)$ & & $69(64)$ & $38(36)$ & \\
\hline 50 and over & $58(51)$ & $55(49)$ & & $6(43)$ & $8(57)$ & \\
\hline BMI-Asian cut offs & & & 0.41 & & & 0.27 \\
\hline Underweight $(\leq 18.49)$ & $5(38)$ & $8(62)$ & & $48(54)$ & $40(46)$ & \\
\hline Normal (18.5-22.9) & $84(47)$ & $95(53)$ & & $176(51)$ & 171(49) & \\
\hline At risk (23.0-24.9) & $79(51)$ & $76(49)$ & & $58(53)$ & $52(47)$ & \\
\hline Obese I (25.00-29.9) & $189(53)$ & $166(47)$ & & $45(51)$ & $43(49)$ & \\
\hline Obese II $(\geq 30.0)$ & $74(46)$ & $87(54)$ & & $10(83)$ & $2(17)$ & \\
\hline Income & & & 0.02 & & & 0.10 \\
\hline$\leq 10,000$ & $147(44.0)$ & $187(56)$ & & $197(49)$ & $203(51)$ & \\
\hline $10,001-20,000$ & $161(55)$ & $134(45)$ & & $92(59)$ & $65(41)$ & \\
\hline$\geq 20,001$ & $124(54)$ & $106(46)$ & & $46(57)$ & $35(43)$ & \\
\hline Education level & & & 0.13 & & & 0.57 \\
\hline Junior high school & $18(53)$ & $16(47)$ & & $5(38)$ & $8(62)$ & \\
\hline High school & 164(49) & $173(51)$ & & $129(50)$ & $127(50)$ & \\
\hline Diploma/certificate & $100(46)$ & $119(54)$ & & $101(53)$ & $89(47)$ & \\
\hline university & $158(56)$ & $126(44)$ & & $104(55)$ & $85(45)$ & \\
\hline
\end{tabular}

Note. *May not total to $\mathrm{N}$ due to missing responses for some characteristics.

${ }^{\dagger} \chi^{2}$ test comparing baseline characteristics between physician interview participants and non-participants for questionnaire self-reported cases

$\chi^{2}$ test comparing baseline characteristics between physician interview participants and non-participants for questionnaire self-reported non- cases 
Table 2. Proportion of self-reported diabetes cases confirmed by physician interviews among participants according to baseline characteristics

\begin{tabular}{|c|c|c|c|c|c|c|c|}
\hline \multirow[b]{2}{*}{$\begin{array}{l}\text { Baseline } \\
\text { characteristics }\end{array}$} & \multirow[b]{2}{*}{$\begin{array}{l}\text { All cases } \\
\text { interviewed } \\
\mathrm{n}\end{array}$} & \multicolumn{3}{|c|}{ All self-reported diabetes cases } & \multicolumn{3}{|c|}{ Self-reported cases with T2DM } \\
\hline & & $\begin{array}{l}\text { Cases } \\
\text { confirmed } \\
\text { during } \\
\text { interview } \\
\mathrm{n}\end{array}$ & $\begin{array}{l}\text { Percent of } \\
\text { cases } \\
\text { confirmed } \\
\%\end{array}$ & $P^{\dagger}$ & $\begin{array}{l}\text { Cases } \\
\text { confirmed } \\
\text { during } \\
\text { interview } \\
\mathrm{n}\end{array}$ & $\begin{array}{l}\text { Percent of } \\
\text { cases } \\
\text { confirmed } \\
\%\end{array}$ & $P^{t}$ \\
\hline Overall & 442 & 364 & $82 \%$ & & 344 & $78 \%$ & \\
\hline Sex & & & & 0.95 & & & 0.006 \\
\hline Males & 282 & 232 & $82 \%$ & & 231 & $82 \%$ & \\
\hline Females & 160 & 132 & $83 \%$ & & 113 & $71 \%$ & \\
\hline Age & & & & 0.13 & & & 0.001 \\
\hline Under 40 & 226 & 180 & $80 \%$ & & 162 & $72 \%$ & \\
\hline 40 or over & 216 & 184 & $85 \%$ & & 182 & $84 \%$ & \\
\hline Income & & & & 0.86 & & & 0.34 \\
\hline 10000 and under & 147 & 123 & $84 \%$ & & 110 & $75 \%$ & \\
\hline $10001-20000$ & 161 & 131 & $81 \%$ & & 125 & $78 \%$ & \\
\hline 20001 and over & 124 & 103 & $83 \%$ & & 102 & $82 \%$ & \\
\hline Education & & & & 0.78 & & & 0.21 \\
\hline Junior high school & 18 & 15 & $83 \%$ & & 15 & $83 \%$ & \\
\hline High school & 164 & 137 & $84 \%$ & & 132 & $80 \%$ & \\
\hline Diploma/certificate & 100 & 84 & $84 \%$ & & 81 & $81 \%$ & \\
\hline University degree & 158 & 126 & $80 \%$ & & 114 & $72 \%$ & \\
\hline
\end{tabular}

Note. ${ }^{\dagger} \chi^{2}$ test comparing the proportion of confirmed self-reported diabetes cases (type 2 diabetes and transient diabetes) by baseline socio-demographic characteristics.

$\$ \chi^{2}$ test comparing the proportion of confirmed self-reported type 2 diabetes cases by baseline socio-demographic characteristics.

The findings from the logistic regression analyses are shown in Table 3. All of the self-reported non-cases correctly reported not having diabetes. Therefore, there was no variability in the socio-demographic characteristics associated with the correct reporting of diabetes status in this group. Among the confirmed self-reported T2DM diabetes cases, the adjusted model shows that female sex is associated with lower odds of agreement between the questionnaire and physician interviews (OR $0.5(95 \%$ CI 0.3-0.9) and that older age is associated with higher odds of agreement between the questionnaire and physician interviews (OR 1.8 (95\% CI 1.1-3.1). When the total group of confirmed self-reported diabetes cases (all types) was included, the association with sex was not apparent and was not statistically significant for age, indicating that these slight differences in the proportion of confirmed cases of T2DM may reflect the cases of gestational diabetes among the young women in this cohort.

Assessing validity of self-report according to sequential reporting pattern over the 2009 and 2013 follow-up surveys showed that the proportion of confirmed T2DM was highest among those who self-reported incident diabetes in both 2009 and 2013 96\% (95\% CI 93-99\%), followed by those who reported incident diabetes only in $201386 \%$ (95\% CI 81-91\%), with the lowest proportion of confirmed T2DM cases being recorded among the participants who reported incident diabetes in 2009 and subsequently no diabetes in 2013 32\% (95\% CI 23-41\%). The group of participants who reported incident diabetes in 2009 and subsequently reported no diabetes in 2013 had the largest proportion of the participants with transient diabetes in this cohort (16 of the total 20 participants with transient diabetes were in this reporting group).

Cumulative incidence of T2DM accounting for the effect of misclassification: Using self-reported questionnaire 
data, the four-year cumulative incidence for T2DM from 2005 to 2009 in the TCS was $1.04 \%$ (95\% CI 0.96-1.12\%; $623 / 59,835)$. However, our physician interviews suggested that $29 \%$ of the group who reported diabetes in 2009 , did not have T2DM. We applied this error to all of the 2009 self-reported cases and calculated a corrected estimate of the 2005 to 2009 four year T2DM cumulative incidence of $0.74 \%$ (95\% CI $0.67-0.81 \%$; 444/59,835). The 2009 to 2013 four-year cumulative incidence of T2DM based on self-report, was 1.05\% (95\% CI 0.97-1.13\%; $411 / 39,220$ ). For this reporting period our physician interviews indicated that, $14.4 \%$ of positive reporters did not have T2DM. By applying this T2DM reporting error to all of the 2013 self-reported cases we calculated a corrected estimate of the 2009 to 2013 four year T2DM cumulative incidence of $0.90 \%$ ( $95 \%$ CI $0.82-0.98 \%$; $352 / 39,220$ ). In a sensitivity analysis we additionally allowed the reporting error fractions to vary from $10-50 \%$ in the group lost to follow-up after 2009 and found that the four year 2005 to 2009 cumulative incidence estimates varied from $0.69 \%(95 \% \mathrm{CI} 0.61-0.75 \% ; 411 / 59,835)$ to $0.79 \%(95 \%$ CI $0.72-0.86 \% ; 473 / 59,835)$.

Table 3. Associations between baseline characteristics and agreement between questionnaire data and interview findings for interviewed self-reported cases

\begin{tabular}{|c|c|c|c|c|c|c|c|c|}
\hline \multirow{3}{*}{$\begin{array}{l}\mathrm{N}=442 \\
\text { Characteristics }\end{array}$} & \multicolumn{4}{|c|}{$\begin{array}{l}\text { All self-reported diabetes cases (Agreed } \\
\text { cases } n=364 \text { ) }\end{array}$} & \multicolumn{4}{|c|}{$\begin{array}{l}\text { Self-reported cases with type } 2 \text { diabetes (Agreed } \\
\text { cases } n=344 \text { ) }\end{array}$} \\
\hline & \multicolumn{2}{|c|}{ Crude } & \multicolumn{2}{|c|}{ Adjusted* } & \multicolumn{2}{|c|}{ Crude } & \multicolumn{2}{|c|}{ Adjusted* } \\
\hline & OR & $95 \% \mathrm{CI}$ & OR & $95 \% \mathrm{CI}$ & OR & $95 \% \mathrm{CI}$ & OR & $95 \% \mathrm{CI}$ \\
\hline \multicolumn{9}{|l|}{ Sex } \\
\hline Male sex & 1 & & 1 & & 1 & & 1 & \\
\hline Female sex & 1.0 & $0.6-1.7$ & 1.0 & $0.6-1.6$ & 0.5 & $0.3-0.8$ & 0.5 & $0.3-0.9$ \\
\hline \multicolumn{9}{|l|}{ Age } \\
\hline Age $<40$ & 1 & & 1 & & 1 & & 1 & \\
\hline Age $\geq 40$ & 1.5 & $0.9-2.4$ & 1.5 & $0.9-2.7$ & 2.1 & $1.3-3.4$ & 1.8 & $1.1-3.1$ \\
\hline $\begin{array}{l}\text { Income per } \\
\text { month }\end{array}$ & & & & & & & & \\
\hline$\leq 10001$ & 1 & & 1 & & 1 & & 1 & \\
\hline $\begin{array}{l}10001-20000 \\
\text { baht }\end{array}$ & 0.9 & $0.5-1.5$ & 0.8 & $0.4-1.5$ & 1.2 & $0.7-2.0$ & 1.0 & $0.6-1.8$ \\
\hline$\geq 20001 \mathrm{baht}$ & 1.0 & $0.5-1.8$ & 0.8 & $0.4-1.7$ & 1.6 & $0.9-2.8$ & 1.1 & $0.5-2.3$ \\
\hline \multicolumn{9}{|l|}{ Education } \\
\hline High school & 1 & & 1 & & 1 & & 1 & \\
\hline Junior high & 1.0 & $0.3-3.6$ & 1.3 & $0.3-5.9$ & 1.2 & $0.3-4.4$ & 1.4 & $0.3-6.8$ \\
\hline Diploma & 1.0 & $0.5-2.0$ & 1.1 & $0.6-2.3$ & 1.0 & $0.5-1.9$ & 1.2 & $0.6-2.4$ \\
\hline University level & 0.8 & $0.4-1.4$ & 0.8 & $0.5-1.5$ & 0.6 & $0.4-1.1$ & 0.6 & $0.4-1.1$ \\
\hline
\end{tabular}

Note. ${ }^{*}$ Results are adjusted for all variables included in the model

95\% CI Confidence Intervals.

\section{Discussion}

This population-based study shows high validity of questionnaire self-reported doctor-diagnosed incident T2DM in younger and middle-aged Thai adults participating in a national cohort study. Using physician interviews as the gold standard, $78 \%$ of self-reported diabetes cases were confirmed as having diagnosed T2DM. Accuracy of self-report did not vary substantially by socio-demographic characteristics in this group of adult students. The proportion of confirmed self-reported cases was slightly lower among the young women in this cohort, a finding that is mostly likely attributed to transient diabetes. These findings highlight the need for cautious interpretation of self-reported diabetes data from a cohort with young women who may be reporting gestational diabetes rather than T2DM. 
We also found that although questionnaire self-reports slightly over-estimated the cumulative incidence of T2DM over one wave of data collection, the misclassification of self-report became negligible once two waves of self-reported data were considered. Therefore, the repeated follow-up of self-reported data essentially eliminates the need for further validity testing of such individuals; a common finding when using repeated measures to assess the validity of self-report (Barr, Herbstman, Speizer, \& Camargo, 2002).

This study has limitations that should be considered when interpreting the findings. Only $52 \%$ of the selected self-reported non-cases and $50 \%$ of the self-reported cases could be contacted by telephone. As such, those contacted may not be representative of the entire non-case and case group in the TCS and the validity of negative response might be lower than $100 \%$. However, there were no significant differences in the socio-demographic characteristics of those interviewed and those who could not be contacted suggesting that the responses in the contacted group may be similar to those of the non-contactable group.

The method used to confirm self-reports in this study was physician telephone interviews, a method that may not be considered 'gold standard'. Accessing medical records, which may have been a better method of confirming doctor-diagnosed T2DM, was not feasible in this population due to the large-scale nature of this nationally dispersed cohort, and to time and cost constraints. However, the physician who conducted the interviews is an experienced clinician with local knowledge of diabetes diagnosis and treatment pathways so is likely to have elicited accurate histories of diabetes diagnoses.

There is an additional study consideration that should be emphasized. The questions in the surveys did not differentiate between type 1 diabetes mellitus (T1DM), type 2 diabetes mellitus or transient (i.e. gestational or corticosteroid medication induced) diabetes. Therefore, it is possible that some of the self-reported incident diabetes cases may have had type 1 diabetes. However, this is unlikely because in this study we excluded prevalent (reported at baseline) cases of diabetes and the median age of this cohort was much higher than that at which T1DM is generally diagnosed.

Our investigations also do not reflect the likelihood that there are undiagnosed cases in this cohort and accordingly a higher incidence of T2DM among TCS participants. However this is unlikely to be a major problem since the participants are well educated and because diabetes awareness has received a great deal of attention in Thailand since the implementation of the national screening program and the national health coverage scheme in the past decade (Aekplakorn et al., 2011; Prakongsai, Limwattananon, \& Tangcharoensathien, 2009).

The high proportion of validated self-reported cases and non-cases found in this study is similar to findings from studies conducted in health professional cohorts (Field et al., 2001; Hu et al., 2001) and is slightly higher than findings reported from two studies conducted among Asian populations (Goto et al., 2013; $\mathrm{Wu}, \mathrm{Li}, \& \mathrm{Ke}, 2000$ ). These differences might be explained by the differences in the characteristics of our study cohort, which was younger and/or had a higher education level than the cohorts sampled in other Asia-based studies (Goto et al., 2013); these characteristics have been shown to be associated with a higher accuracy of self-reporting diabetes in some prior research (Molenaar et al., 2007; Okura et al., 2004). The association between older age and the inaccurate reporting of chronic diseases has been attributed to declining cognitive function (Sherbourne \& Meredith, 1992), and/or the reluctance of the elderly to admit or perceive that they have a chronic disease (Kriegsman, Penninx, Van Eijk, Boeke, \& Deeg, 1996). Furthermore, among some Asian immigrant groups, younger age has been found to be associated with higher levels of formal education (Tseng, Halperin, Ritholz, \& $\mathrm{Hsu}, 2013)$. Accordingly, the association between age and the inaccurate reporting of health status may partially be explained by education levels (Goldman et al., 2003). The proportion of validated self-reported cases in this study was slightly lower than findings from one study conducted in China. This may be due to the higher prevalence of diabetes in their older study cohort (Yuan et al., 2015).

Although the validity of self-reported doctor diagnosed diabetes in this study was high, these findings may be less applicable to the broader Thai population, which, on average, is older and has a lower education level than the Thai Cohort Study participants (A. C. Sleigh et al., 2008). Nonetheless, despite the differences in the cohort structures and gold standard methods used to validate self-reported diabetes in this study and among other validation studies, the general findings, from both Western populations conducted mostly with older and highly educated cohorts and Asian populations conducted mostly with younger and/or less educated cohorts, are that the validity of survey self-reported diabetes is generally high (Goldman et al., 2003; Huerta, Tormo, Egea-Caparrós, Ortolá-Devesa, \& Navarro, 2009; Wu et al., 2000). Clearly, careful attention must be given to structuring the diagnostic questions, with specification of doctor-diagnosis and (if relevant) hospitalization having shown to be important for many diseases (Barr et al., 2002; Yuan et al., 2015).

Our study found that personal socio-demographic characteristics were not statistically significantly associated 
with the validity of self-reported doctor diagnosed diabetes. The lack of significant differences in accuracy of reporting across personal characteristics and high overall agreement between questionnaire data and physician telephone interviews is likely due to the medical importance of this disease (Goldman et al., 2003). Diabetes requires ongoing regular medical treatment and engagement with medical professionals long after its diagnosis (Kehoe, Wu, Leske, \& Chylack, 1994; Pastorino et al., 2014). Although conditions such as hypertension share some of these same medical qualities, the accuracy of survey self-reported hypertension is generally lower than the accuracy of survey self-reported diabetes. This may be because hypertension can be a less disabling disease than diabetes during everyday life, people who are controlling their hypertension may think that they no longer have the condition and may be less likely to report it (Molenaar et al., 2007). Furthermore, hypertension is not recognized as a chronic disease by some ethnic groups (Goldman et al., 2003).

Data from repeated measures showed that the proportion of confirmed survey self-reports was highest among the participants who self-reported the same diabetes status consistently at the two follow-up questionnaires. Conversely, the proportion of confirmed survey self-report was the lowest among the participants who self-reported incident diabetes in 2009 and subsequently self-reported not having diabetes in 2013. We found that over a third of these participants had transient diabetes (gestational or corticosteroid medication induced) in the first follow-up questionnaire and as such accurately reported not having diabetes in the second follow-up questionnaire. Although transient diabetes generally has a low prevalence in cohort studies, particularly in cohorts that are older than this one, using repeated measures was found to be a good tool for detecting the 'true' T2DM cases. Moreover, having a four year interval in between follow-ups enabled the identification of transient diabetes due to gestational diabetes.

Cumulative incidence estimates calculated using the questionnaire data and the physician interview-corrected data demonstrate that questionnaire self-report slightly over-estimated the cumulative incidence. Although this slight over-estimation should be taken into account when calculating T2DM incidence and its determinants in this cohort, it is likely to have minimal impact on relative risk measures (Copeland, Checkoway, McMichael, \& Holbrook, 1977) (Rothman, Greenland, \& Lash, 2008).

\subsection{Conclusions}

The current study demonstrates that the proportion of questionnaire self-reported doctor-diagnosed incident T2DM cases confirmed using physician interviews is high and that questionnaire T2DM self-report is a valid tool for detecting new cases of T2DM in a large Thai population-based study. These findings suggest that self-reported incident T2DM can be used to assess the trends and determinants of incident T2DM, particularly in younger and educated Thai adults.

\section{Acknowledgements}

This study was supported by the International Collaborative Research Grants Scheme with joint grants from the Wellcome Trust UK (GR071587MA) and the Australian National Health and Medical Research Council (NHMRC, grant No.268055). It was also supported by a global health grant from the NHMRC (585426). SJ has a career development fellowship from the NHMRC. KP has an Australian Postgraduate Award from the Australian National University. We thank the team at Suhkkothai Thammathirat Open University (STOU) who assisted with student contact and the STOU students who are participating in the Thai Cohort Study.

\section{Competing Interests Statement}

The authors declare that there are no conflicts of interest.

\section{Ethics and Consent}

Ethical approval was obtained from Sukhothai Thammathirat Open University Research and Development Institute (protocol 0522/10) and the Australian National University Human Research Ethics Committee (protocol 2004344, 2009570 and 2014/782).

\section{Consent}

The physician received verbal consent to publish all interview data from each interviewee prior to conducting the interview.

\section{Authors' Contributions}

KP devised the validation study, constructed the interview protocol, entered and analysed all of the data and wrote the paper. PT conducted the telephone interviews and assisted in the development of the interview protocol. SJ, CB and AS assisted with the planning of the study and its required analyses, with the development of the 
interview protocol, with the interpretation of the study findings and with the editing of all drafts. CD guided the analytical approach of this paper, supervised all analyses of the data and helped with the interpretation of the study findings. AS and SS conceived and developed the cohort. All authors approved the final manuscript.

\section{References}

Aekplakorn, W., Chariyalertsak, S., Kessomboon, P., Sangthong, R., Inthawong, R., Putwatana, P., \& Taneepanichskul, S. (2011). Prevalence and management of diabetes and metabolic risk factors in Thai adults the Thai national health examination survey IV, 2009. Diabetes Care, 34(9), 1980-1985. https://doi.org/10.2337/dc11-0099

American Diabetes Association. (2010). Diagnosis and classification of diabetes mellitus. Diabetes Care (Vol. 33, pp. S62-S69).

Barr, R. G., Herbstman, J., Speizer, F. E., \& Camargo, C. A. (2002). Validation of self-reported chronic obstructive pulmonary disease in a cohort study of nurses. American Journal of Epidemiology, 155(10), 965-971. https://doi.org/10.1093/aje/155.10.965

Chan, J. C., Malik, V., Jia, W., Kadowaki, T., Yajnik, C. S., Yoon, K.-H., \& Hu, F. B. (2009). Diabetes in Asia: epidemiology, risk factors, and pathophysiology. Jama, 301(20), 2129-2140. https://doi.org/10.1001/jama.2009.726

Chokevivat, V., Chuthaputti, A., \& Khumtrakul, P. (2005). The use of traditional medicine in the Thai health care system. Region consultation on development of Traditional medicine in the South East Asia region, Pyongyang, DPR Korea, 22-24.

Comino, E. J., Tran, D. T., Haas, M., Flack, J., Jalaludin, B., Jorm, L., \& Harris, M. F. (2013). Validating self-report of diabetes use by participants in the 45 and up study: a record linkage study. BMC Health Services Research, 13(1), 481. https://doi.org/10.1186/1472-6963-13-481

Copeland, K. T., Checkoway, H., McMichael, A. J., \& Holbrook, R. H. (1977). Bias due to misclassification in the estimation of relative risk. American Journal of Epidemiology, 105(5), 488-495.

El Fakiri, F., Bruijnzeels, M. A., \& Hoes, A. W. (2007). No evidence for marked ethnic differences in accuracy of self-reported diabetes, hypertension, and hypercholesterolemia. Journal of Clinical Epidemiology, 60(12), 1271-1279. https://doi.org/10.1016/j.jclinepi.2007.02.014

Field, A. E., Coakley, E. H., Must, A., Spadano, J. L., Laird, N., Dietz, W. H., . . Colditz, G. A. (2001). Impact of overweight on the risk of developing common chronic diseases during a 10-year period. Archives of Internal Medicine, 161(13), 1581-1586. https://doi.org/10.1001/archinte.161.13.1581

Goldman, N., Lin, I.-F., Weinstein, M., \& Lin, Y.-H. (2003). Evaluating the quality of self-reports of hypertension $\begin{array}{llll}\text { and diabetes. Journal of Clinical Epidemiology, } & 56(2), & 148-154 .\end{array}$ https://doi.org/10.1016/S0895-4356(02)00580-2

Goto, A., Morita, A., Goto, M., Sasaki, S., Miyachi, M., Aiba, N., . . Watanabe, S. (2013). Validity of Diabetes Self-Reports in the Saku Diabetes Study. Journal of Epidemiology, 23(4), 295. https://doi.org/10.2188/jea.JE20120221

Hesketh, T., \& Zhu, W. X. (1997). Health in China. Traditional Chinese medicine: one country, two systems. BMJ: British Medical Journal, 315(7100), 115. https://doi.org/10.1136/bmj.315.7100.115

Hu, F. B., Leitzmann, M. F., Stampfer, M. J., Colditz, G. A., Willett, W. C., \& Rimm, E. B. (2001). Physical activity and television watching in relation to risk for type 2 diabetes mellitus in men. Archives of Internal Medicine, 161(12), 1542-1548. https://doi.org/10.1001/archinte.161.12.1542

Huerta, J. M., Tormo, M. J., Egea-Caparrós, J. M., Ortolá-Devesa, J. B., \& Navarro, C. (2009). Accuracy of self-reported diabetes, hypertension, and hyperlipidemia in the adult Spanish population. DINO study findings. Revista Española de Cardiología (English Edition), 62(2), 143-152. https://doi.org/10.1016/S1885-5857(09)71532-4

International Diabetes Federation. (2015). IDF Diabetes Atlas. Retrieved from Brussels, Belgium: www.idf.org/diabetesatlas

Kehoe, R., Wu, S.-Y., Leske, M. C., \& Chylack, L. T. (1994). Comparing self-reported and physician-reported medical history. American Journal of Epidemiology, 139(8), 813-818.

Kelly, M., Seubsman, S.-a., Banwell, C., Dixon, J., \& Sleigh, A. (2014). Thailand's food retail transition: 
supermarket and fresh market effects on diet quality and health. British Food Journal, 116(7), 1180-1193. https://doi.org/10.1108/BFJ-08-2013-0210

Kriegsman, D. M., Penninx, B. W., Van Eijk, J. T. M., Boeke, A. J. P., \& Deeg, D. J. (1996). Self-reports and general practitioner information on the presence of chronic diseases in community dwelling elderly: a study on the accuracy of patients' self-reports and on determinants of inaccuracy. Journal of Clinical Epidemiology, 49(12), 1407-1417. https://doi.org/10.1016/S0895-4356(96)00274-0

Manson, J. E., Stampfer, M., Colditz, G., Willett, W., Rosner, B., Hennekens, C., . . Krolewski, A. (1991). Physical activity and incidence of non-insulin-dependent diabetes mellitus in women. The Lancet, 338(8770), 774-778. https://doi.org/10.1016/0140-6736(91)90664-B

Margolis, K. L., Qi, L., Brzyski, R., Bonds, D. E., Howard, B. V., Kempainen, S., . . Tinker, L. T. (2008). Validity of diabetes self-reports in the Women's Health Initiative: comparison with medication inventories and fasting glucose measurements. Clinical Trials, 5(3), 240-247. https://doi.org/10.1177/1740774508091749

Molenaar, E. A., Van Ameijden, E. J., Grobbee, D. E., \& Numans, M. E. (2007). Comparison of routine care self-reported and biometrical data on hypertension and diabetes: Results of the Utrecht Health Project. The European Journal of Public Health, 17(2), 199-205. https://doi.org/10.1093/eurpub/ck1113

Offringa, L. (2014). Medicinal Plants of Northern Thailand for the Treatment of Cognitive Impairment in the Elderly. Cham, Switzerland: Springer.

Okura, Y., Urban, L. H., Mahoney, D. W., Jacobsen, S. J., \& Rodeheffer, R. J. (2004). Agreement between self-report questionnaires and medical record data was substantial for diabetes, hypertension, myocardial infarction and stroke but not for heart failure. Journal of Clinical Epidemiology, 57(10), 1096-1103. https://doi.org/10.1016/j.jclinepi.2004.04.005

Pastorino, S., Richards, M., Hardy, R., Abington, J., Wills, A., Kuh, D., \& Pierce, M. (2014). Validation of self-reported diagnosis of diabetes in the 1946 British birth cohort. Primary care diabetes, 9(5).

Pradhan, A. D., Manson, J. E., Rifai, N., Buring, J. E., \& Ridker, P. M. (2001). C-reactive protein, interleukin 6, and risk of developing type 2 diabetes mellitus. Jama, 286(3), 327-334. https://doi.org/10.1001/jama.286.3.327

Prakongsai, P., Limwattananon, S., \& Tangcharoensathien, V. (2009). The equity impact of the universal coverage policy: lessons from Thailand. Adv Health Econ Health Serv Res, 21, 57-81. https://doi.org/10.1108/S0731-2199(2009)0000021006

Ramachandran, A., Wan Ma, R. C., \& Snehalatha, C. (2010). Diabetes in Asia. The Lancet, 375(9712), 408-418. https://doi.org/10.1016/S0140-6736(09)60937-5

Rothman, K. J., Greenland, S., \& Lash, T. L. (2008). Modern Epidemiology (3rd ed.). Philadelphia: Wolters Kluwer Health/Lippincott Williams \& Wilkins.

Rylander, C., Sandanger, T. M., Engeset, D., \& Lund, E. (2014). Consumption of lean fish reduces the risk of type 2 diabetes mellitus: A prospective population based cohort study of Norwegian women. PloS One, 9(2), e89845. https://doi.org/10.1371/journal.pone.0089845

Shaw, J. E., Sicree, R. A., \& Zimmet, P. Z. (2010). Global estimates of the prevalence of diabetes for 2010 and 2030. Diabetes Research and Clinical Practice, 87(1), 4-14. https://doi.org/10.1016/j.diabres.2009.10.007

Sherbourne, C. D., \& Meredith, L. S. (1992). Quality of self-report data: a comparison of older and younger chronically ill patients. Journal of Gerontology, 47(4), S204-S211. https://doi.org/10.1093/geronj/47.4.S204

Sleigh, A., \& Seubsman, S. (2015). Studying the Thai Health-Risk Transition. In Butler Colin, J. Dixon, \& A. Capon (Eds.), Healthy People, Places and Planet (pp. 166-176). Canberra: ANU Press.

Sleigh, A. C., Seubsman, S., Bain, C., \& The Thai Cohort Study Team. (2008). Cohort Profile: The Thai Cohort of 87134 Open University students. International Journal of Epidemiology, 37(2), 266-272. https://doi.org/10.1093/ije/dym161

Tseng, J., Halperin, L., Ritholz, M. D., \& Hsu, W. C. (2013). Perceptions and management of psychosocial factors affecting type 2 diabetes mellitus in Chinese Americans. Journal of Diabetes and its Complications, 27(4), 383-390. https://doi.org/10.1016/j.jdiacomp.2013.01.001

Wu, S.-C., Li, C., \& Ke, D. (2000). The agreement between self-reporting and clinical diagnosis for selected medical conditions among the elderly in Taiwan. Public Health, 114(2), 137-142. 
https://doi.org/10.1016/S0033-3506(00)00323-1

Yuan, X., Liu, T., Wu, L., Zou, Z.-Y., \& Li, C. (2015). Validity of self-reported diabetes among middle-aged and older Chinese adults: the China Health and Retirement Longitudinal Study. BMJ Open, 5(4), e006633. https://doi.org/10.1136/bmjopen-2014-006633

\section{Copyrights}

Copyright for this article is retained by the author(s), with first publication rights granted to the journal.

This is an open-access article distributed under the terms and conditions of the Creative Commons Attribution license (http://creativecommons.org/licenses/by/4.0/). 